\section{Cureus}

\title{
The Stem Cell Vascular Niche in Brain Tumorigenesis
}

\author{
Victor Tse $^{1}$, Harish Babu ${ }^{2}$ \\ 1. Department of Neurosurgery, Kaiser Permanente, Redwood City, California 94063 2. Department of \\ Neurosurgery, Institute for Stem Cell Biology and Regenerative Medicine, Stanford University
}

$\square$ Corresponding author: Victor Tse, tsevictor@gmail.com

Disclosures can be found in Additional Information at the end of the article

\section{Abstract}

The cancer stem cell vascular niche is a physical and biological unit composed of a conglomerate of innate cancer stem cells and progenitor cells that are recruited into this dynamic and highly complex milieu. Collectively they modify the extracellular matrix and form a permissible microenvironment to facilitate the renewal, proliferation, invasion, and differentiation of the cancer stem cells, and those of the auxiliary cells. The angioarchitecture within the tumor is grossly abnormal as these vessels are of varying caliber and arborization. These abnormal tumor vessels can be generated from endothelial progenitor, hematopoietic, monocytic and mesenchymal stem cells derived from the bone marrow (BM). Recent studies have observed that tumor endothelium is also derived from cancer stem cells transforming our understanding of the intimate relationship between cancer stem cells and vascular niche. Given these observations, it is conceivable that mutation at the level of vasculature may translate to an abnormal vascular niche discharging unregulated stem cell signals and thus propel aberrant cancer stem cells to achieve their lethal malignant potential. In this review we summarize the cellular and molecular components of stem cell vascular niche with a special emphasis on brain tumors.

Categories: Neurosurgery, Oncology

Keywords: vascular niche, glioma, neural stem cell, cancer stem cell, angiogenesis, endothelium

\section{Introduction And Background}

The idea of cancer initiating cells associated within the tumor-angioarchitecture was first mentioned in the monograph, A classification of tumors by Bailey and Cushing in 1926 [1]. They described the unipolar astroblasts whose "tails become attached to the walls of capillaries". The temporal-causal relationship of vascular development and tumorigenesis was indisputably demonstrated by Folkman, and refined by Jain and others [2-3]. In late 1990s, Holash, et al. suggested that dormant tumor cells commonly resided in the proximity of blood vessels, and as the tumor expands they "co-opt" new vessels toward themselves [4]. The glioblastoma (GBM)

Published 09/18/2011

\section{Copyright 2011}

Tse et al. This is an open access article distributed under the terms of the Creative Commons Attribution License CC-BY 3.0., which permits unrestricted use, distribution, and reproduction in any medium, provided the original author and source are credited. vessels are tortuous, highly permeable and characterized by abnormalities in their cellular components and basement membrane. These vessels are used as a means to support their needs and as conduit along which they invade and proliferate. These work underpinned the functional relevance of tumor vascular niche. Recently Gilberston and others [5] have shown that brain cancer initiating cells - the cancer stem cells (CSC) are closely located with preexisting vascular trees. Together they re-shape the neo-angioarchitecture and by encompassing auxiliary cells and their surround basal-matrix, they form the vascular niche complex where cancer stem cells evolve and propagate. It is the cellular activity within these complexes that dictate the robustness of tumorigenesis. It is plausible that within these niches cancer stem 
cells remain in a steady state between proliferation and quiescence prior to further expansion and invasion, hence the dormant phase of tumorigenesis.

\section{Review}

\section{The cancer stem cell hypothesis}

There is a striking resemblance between neural stem cell biology and glioma biology. Both can be identified with similar markers, such as nestin and CD133 [6-7]. In the case of the neural stem cell, the balance between proliferation and differentiation is highly regulated. While in glioma, there is marked dys-regulation of the balance between proliferation and differentiation, resulting in uncontrolled self-renewal and associated tumor growth and incomplete differentiation (Table 1). Recent studies have suggested tumors initiate from cells that harbor chromosomal defects that predispose themselves to further genomic mutation. Accumulated genomic defects eventually reach a threshold where they decouple from physiological cell-cycle regulatory checkpoints and undergo unchecked proliferation [8-9]. Within those cluster of mutated cells lies a subpopulation of tumorigenic cells that shares a number of characteristic similar to stem cells, characters such as the ability to self-renewal, proliferate, invade, and differentiate. This population of "stem-like" cells within the tumor may serve as the reservoir for future cancer-initiating cells. Furthermore, the open chromosomal structure observed in these cells exposes them to the risk of accumulating deleterious mutations over the lifetime of the organism. This accumulation of aberrant genetic changes has been suggested to be the principle cause of cancer formation [10]. This hypothesis suggests that expansion of transformed stem-like cells (cancer stem cells) replenishes the tumorigenic cancer cells that perpetuate the growth of the tumor. Singh, et al. have provided experimental evidence in the role of this sub-population of cells in the initiation of GBM, the most devastating of the brain tumors [11]. Subsequent studies have suggested that this cancer stem cell population may undergo aberrant differentiation in addition to genetic instability and epigenetic change, resulting in cellular heterogeneity that is commonly observed within the tumor mass [12-13]. 


\section{Cureus}

\section{Neural Stem Cells Vascular Niche}

Anatomical Restricted to dentate gyrus and Subventricular

location

Zone

Epoch

Cellular

contribution

Presents normally during development and adult neurogenic niches

Minor contribution of vascular endothelium from neural stem cells. Primarily from progenitors of endothalial and its associated axuillary cells

Genetical stability

Vessel architecture

Normal vessels with normal tissue oxygen

Migration
Genetic abnormality is absent

delivery.

No abnormal migration

\section{Cancer Stem Cell Vascular Niche}

Any region occupying tumor

Present only in pathological state of glioma.

Possible contribution of vascular endothelium from cancer stem cells, in addition to progenitors of endothalial and its associated axuillary cells

Significant genetic and epigenetic mutation

Vessels are highly tortuous and present with tissue hypoxia

Prolific migration and infiltrates normal tissue.

\section{TABLE 1: Difference in vascular niche between normal neural stem cells and cancer}

stem cells

If cancer is viewed as developmental cues gone awry, then it is not surprising that cancer and stem cells share many molecular mechanisms mediating important cellular processes such as self-renewal, differentiation, and possible "fate determined" migration [14]. Furthermore, tumors exhibit a cellular hierarchy similar to that found during normal development [10]. This hierarchy allows tumors to recruit new blood vessels and coax other cell types to the tumor milieu. This system functions independently of the normal physiologically regulated systems (Figure 1). 


\section{Cureus}
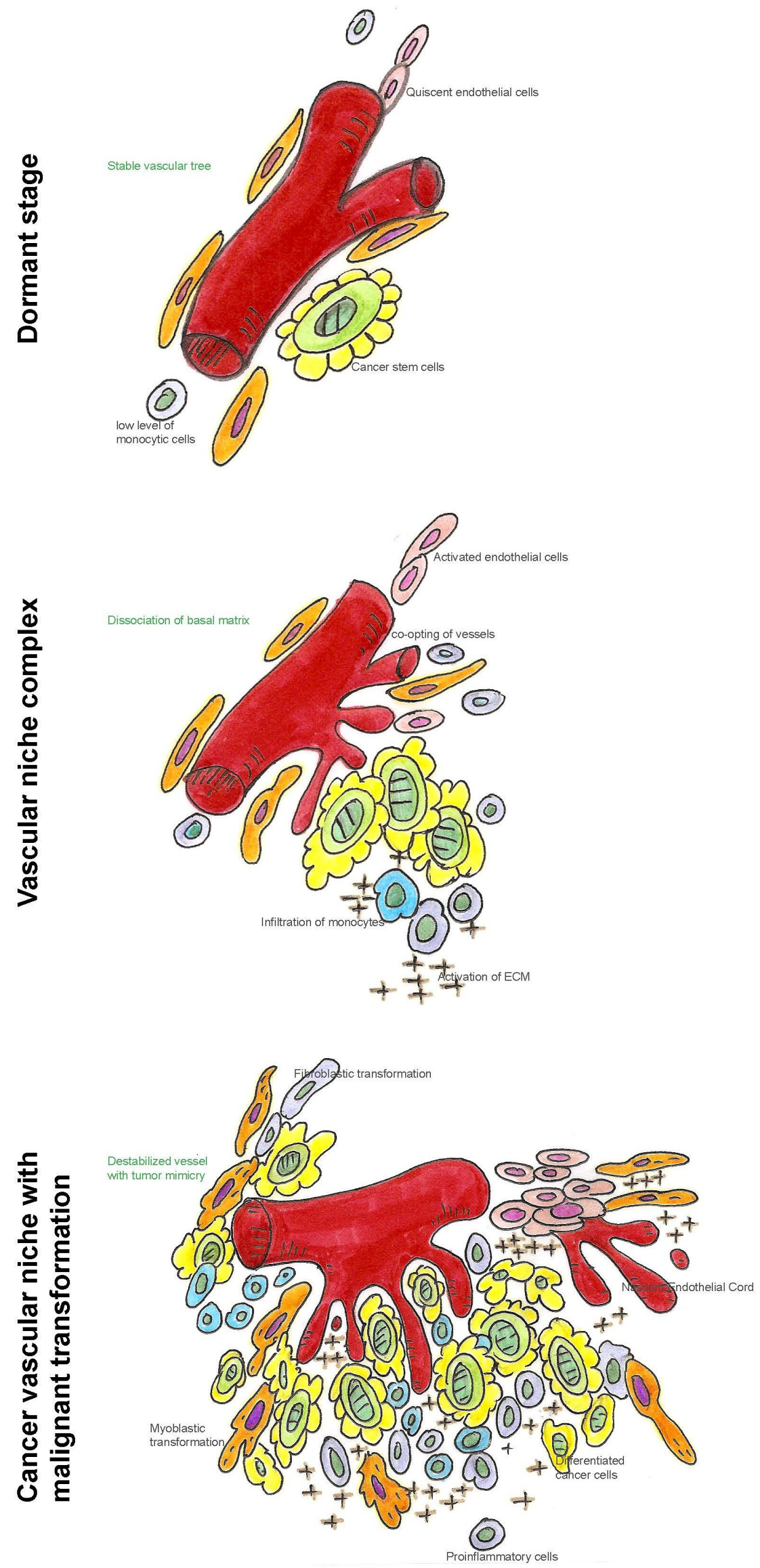

FIGURE 1: Schematic representation of proposed model for 


\section{stages of gliomagenesis}

Dormant stage Cancer stem cells destined to become glioma are present adjacent to blood vessels. The endothelial cells are quiescent at this stage and the tumor milieu contains low levels of monocytic cells. With increased proliferation of cancer stem cells there is an increased infiltration of monocytes. This is also associated with activation of endothelial cells. With the accompanying breakdown of ECM, the endothelial cells form nascent endothelial cord. The generation of reactive oxygen and nitrogen species aids this process

\section{The cellular constituency of the cancer stem cell-vascular niche complex}

Calabrese and colleagues have reported that there is a population of Nestin ${ }^{+} / \mathrm{CD} 133^{+}$in brain tumor, which is located in areas of increased micro-vessel density [15]. These vessel-associated cancer stem cells were in direct contact with the capillary networks, whereas CD133- cancer cells were diffusely distributed with no apparent vascular localization [16]. Moreover, CD133 tumor cells were shown to home towards endothelial vascular tubes and initiate contact along the entire length of exposed vasculature. The location and microenvironment dependence of these initiating glioma stem cells have also been confirmed in other tumors [17]. In addition to these $\mathrm{CD} 133^{+}$cells within the tumor, there exists endothelial progenitors and bone marrow (BM) derived cells. A number of animal models have suggested the contribution of endothelial progenitor and BM-derived cells in tumor neo-vascularization [18-19]. This supports the notion that vasculogenesis is an important epoch in tumor growth.

\section{The role of endothelial progenitor cells in the formation of the vascular niche}

It is widely believed that circulating endothelial progenitor cells and bone marrow-derived progenitors home into the site of robust neo-vascularization. This is despite the fact that purified populations of EPC has been challenging to isolate and culture as homogenous populations. Though prominent markers such as CD133 and KDR are expressed by endothelial progenitor cells, these markers are non-specific and label several cells types outside of the classified EPC subtype. Once arriving at the tissue site, under the influence of local VEGF and PDGF, these circulating progenitor cells dock onto the "co-opting" blood vessels, vessels that have partially or completely dissociated basal matrix mediated by E and P selectins [20-21]. The ensuing extravasation forms the initial step in the sprouting of new vessels within the tumor and in their periphery [22]. The evidence for the contribution of BM-derived endothelial progenitor cells (EPCs) in tumor angiogenesis is relatively well-established [23-24]. However, the ontogenesis of these EPCs is still debatable. Most endothelial progenitor cells do not differentiate into mature endothelial cells but may rather affect vasculogenesis by releasing of proangiogenic cytokines. It is plausible that BM derived EPCs are derived from putative hemangioblasts which are the precursors of both hematopoietic and endothelial cell lineages. These hemangioblasts express phenotypic markers of hematopoietic stem cells (HSCs) [25] as well as differentiated endothelial cells [26-27] and are thus antigenically difficult to distinguish from EPCs since both express CD133, a stem cell's marker. Moreover, both cell types may have resided in the same location within the adult bone marrow. Once in peripheral blood, they home to sites of tissue ischemia, trauma, or tumor growth, where they differentiate into endothelial cells to support vascular remodeling [28-30]. Studies in various solid tumors have revealed that EPCs differentiate into mature ECs, and these ECs are incorporated into the lumen of emerging neovasculature [31].

\section{The role of BM-derived cells in the vascular niche formation}


BM-derived mesenchymal stem cells (MSCs) also play an important role in post-natal vasculogenesis, notably during tissue ischemia and tumor vascularization. Their primary function is related to the formation of the perivascular cells and the associated matrix. These putative perivascular progenitor cells are recruited by PDGF-stimulated VEGF expression in tumor endothelial cells to support and stabilize the nascent vessels [32]. Although these pericytic cells are loosely associated with tumor blood vessels and reduced in number when compared with normal tissue vasculature [33], they appear to be important cell constituents in tumor angiogenesis [34]. These pericytic cells contribute to the early phase of angiogenic sprouting, tube formation, and survival of the vascular niche within tumors [35]. They are cells that guide the endothelial cell cord at the advancing edge of neovascular formation, and later on they secure the integrity of the nascent vessels. Systemically administered MSCs target sites of growing tumors [36]. Congruent with the hypoxic conditions within gliomas, an associated rise in HIF-1a expression leads to recruitment of BM-derived MSC to promote neovascularization in GBM via interplay between MMP9 and VEGF [20]. Although MSCs do not express VEGF receptors, Ball, et al. recently identified that VEGF-A can stimulate PDGF receptors, which in turn regulates MSC migration and proliferation [37]. Recent evidences suggest that MSCs may even be the progenitor cells from which fibroblasts within the tumor stroma are derived [38].

Monocytes and fibroblastic cells in the formation of CSC-vascular niche complex

There is a temporal window just prior to the expansion of the tumor mass which enables the incorporation of BM-derived progenitor cells into the tumor vessel-wall [39,40]. Within this window of time, the recruited cells generate a conducive microvascular environment. At that juncture, the pre-existing vessel wall within this defined space serves as an independent niche for the recruitment and insertion of EPCs, MSCs and other monocytic progenitors cells. The recruitment of these progenitor cells by CSCs helps in the establishment of a nascent vascular niche for tumorigenesis. $\mathrm{CD} 11^{+}$circulating monocytes recruited into the vascular niche acquire an endothelial-like phenotype and are incorporated both into the lumen and perivascular space. These monocytic cells provide "reconstitute and instructive" signals for tumor establishment $[41,42]$.

An emerging hypothesis states that CSCs drive tumorigenesis by directly inducing an inflammatory phenotype within the cancer stem cell-vascular niche complex, thus catapulting a conductive to an inductive environment [43]. This occurs by recruiting immunocytes and promoting stromal remodeling as seen in aberrant stem cell-vascular niche that contributes to myeloproliferative diseases [44]. The migration of hematopoetic pro-angiogenic cells, most notably macrophages and mast cells, aids in the production of pro-angiogenic factors, proteases, and growth factors responsible for the creation of a microenvironment that stimulates epithelial-cell migration, survival, and proliferation. Of particular importance are monocytic cells such as macrophages, a key component in the cellular constituent of inflammation. In inflammatory tissues, macrophages are predominantly of the M1 phenotype. These cells produce high levels of reactive oxidation species (ROS) and inflammatory cytokines, resulting in potent antimicrobial, immuno-stimulatory and tumor cytotoxic functions. In response to tumor-derived cytokines these macrophages acquire the M2 phenotype [45]. They are a potent source of the mediators that perpetuate the inflammatory process, and they release reactive oxygen and nitrogen species. ROS have also been shown to modify the activity of myeloid-derived suppressor cells (MDSCs). MDSCs are directly involved in tumor angiogenesis; they stimulate angiogenesis and ECM breakdown through the production of angiogenic growth factors and MMPs. Secretion of MMPs and other proteinases by macrophages in the vascular niche enhance cancer-cell motility, dispersion and invasion. The reactive oxygen and nitrogen molecules can directly damage DNA and modify the proteins that are involved in DNA repair. Moreover, MDSCs inhibit anti-tumor immunity. In combination with nitric oxide, MDSC- 
derived ROS contribute to the generation of peroxynitrite [46]. The latter causes the nitration of various tyrosines on proteins, including the T-cell receptor CD8. This modification alters antigen recognition and thereby induces T-cell tolerance [47].

In addition to monocytic cells there is influx of fibroblasts, and some of the monocytic cells may undergo epigenetic transformation with fibroblastic/myofibroblastic morphology [48,49]. These cells produce chemokine such as stromal-cell-derived factor1 (SDF1), a mitogen in its own right. Myofibroblast-derived SDF1 promotes the proliferation of mammary carcinoma cells that express its high-affinity receptor CXCR4, and likely contributes to cancer growth through the regulation of angiogenesis by attracting endothelial progenitor cells [50]. Being highly mobile, fibroblasts form the advancing edge of the tumor with the nascent vascular cord in tow. The cancer cells will adhere along these conduits to spread to the surrounding normal tissue ignoring tissue boundary and autonomy that have partly destroyed or interrupted during matrix modification [51].

\section{Cancer stem cells derived endothelium}

Recently it has become evident that glioma stem cells have cell lineage potential beyond what was conventionally assigned to them. $\mathrm{CD} 133^{+}$glioma stem cells that carry chromosomal abnormalities also generate genetically aberrant endothelial cells that profoundly contribute to the vascular architecture of the glioma [52,53]. Such tumor derived endothelial cells produce VEGF and have the potential to generate highly vascularized anaplastic tumors. When the GBM-derived endothelium was genetically targeted and killed, the entire tumor became necrotic suggesting the vital role the new born endothelium play in the survival of tumor. These studies also highlighted the crucial role of Notch in the transition of tumor stem cells into tumor endothelial cells. It is noteworthy that Notch has been shown to play an important role in modulating cancer stem cell renewal and proliferation. Mutation in Notch leads to uncontrolled proliferation and renewal of cancer stem cells [54].

\section{Molecular regulators of CSC-Vascular Niche Complexes}

Hypoxia and its associated signal transduction pathways: Most cells have to be within 100-200 $\mu \mathrm{m}$ from its blood supply to receive adequate nutrition. Beyond this distance there is a precipitous drop in $\mathrm{O}_{2}$ diffusion efficiency. Tumors are known to utilize glycolysis to generate lactate from glucose even in the presence of abundant oxygen, a phenomenon known as Warburg effect [55]. This potentially serves two purposes, first it enables the tumor cells to generate intermediates for cells growth and division [56]; second by relying less on mitochondria for ATP generation, and there is high mitochondrial membrane potential conferring apoptosis resistance [57]. These inhibited mitochondria in tumor cells transmit pseudo hypoxic redox signals and activate HIF-1a even during normoxia [58]. They are coordinately regulated by oncogenes such as PI3K-Akt and Myc [59]. The excess lactate generate by this aerobic glycolysis also promotes angiogenesis and matrix breakdown, events that facilitate metastasis [60]. Glycolysis also leads to decreased production of a-ketoglutarate, a direct product of Krebs cycle, which may also promote HIF-1a activation because it is a cofactor for the prolyl hydroxylation reaction that degrades HIF-1a [58]. In a study by Michelakis et al, administration of dichloroacetate, a drug that forces pyruvate to enter Krebs cycle significantly inhibited glioma and is in clinical trial [61]. Both in vivo and in vitro, Dichloroacetate inhibited HIF-1a as well as suppressed angiogenesis.

Tumor blood flow not only varies spatially and temporally during tumor growth but it also changes according to the state of its angioarchitecture [62]. It is particularly true for vessels that are within the inner mass of the tumor, where the vessels are chaotic and sinusoidal in 
appearance and the blood flow is non-laminar and may even be stagnant in some part of its course [63]. Hypoxia induced expression of HIF-1a leads to increase expansion [64] and tumorigenic potential [65] of CD $133^{+}$glioma stem cells which could lead to increased tumor derived neo-angiogenesis [52,53]. The expression of HIF-1a and VEGF are relatively robust in the region of relative hypoxia and in the presence of Angiopoietin -2, they signal endothelial proliferation, basal laminar fragmentation, and facilitate sprouting $[65,66]$. Angiopoietin-1 recruits perivascular/mural cells, which stabilizes nascent vessels and maintains their integrity; however, the expression of Angiopoietin-1 changes during the course of tumor expansion. The exact etiology of the preferential spatial/ temporal expression of these factors remains unclear. This altering expression could potentially be the cause of involution and dysmorphic transformation of the neovascular tree. The hostile microenvironment with low $\mathrm{pH}$, low partial $\mathrm{O}_{2}$ pressure and necrotic tissue sustains tumor progression and potentially drug resistance. It is particular true in the "older" central core of the tumor [4,67], a region routinely associated with tumor necrosis. It is plausible that the confounding effects of low oxygen tension combined with dysmorphic vascular tree favors cancer stem cells differentiation as opposed to cancer stem cell renewal.

Cancer stem cells cultured in the presence of vascular endothelial cells showed a five-fold increase in growth as well as maintenance of aggregated tumor spheres and self-renewal capacity [15]. These features were absent in those cells cultured without endothelial cells or their associated signaling factors. In vivo, cells that are associated with the cancer stem cell niche, influence the fate of all neighboring cell types including naïve neuronal cells. The multidirectional regulatory process within this angio-architecture is orchestrated by stem cell related trophic and angiogenic factors such as members of the Wnt, Sonic Hedgehog (SHH) and TGF- $\beta$ family, and also the intercellular physical contact. These contacts interact with anchoring molecules within the ECM such as VCAM-1/VLA-4, fibronectin, heparans, and other integrin [68-71]. ECM receptors such as integrin-a6 expressed on GBM cancer stem cells promote their maintenance [72]. When these integrins on cancer stem cells are targeted, there is a decrease in self-renewal, proliferation and tumor forming capacity. Indeed cilengitide, an inhibitor of integrins $\mathrm{a}_{\mathrm{v}} \mathrm{b} 3$ and $\mathrm{a}_{\mathrm{v}} \mathrm{b} 5$ (which are in clinical trials) have shown good response in preclinical trials [73]. Additionally, some of the factors and proteins sequestered within the cellular matrix are liberated by the proteinases and trigger intracellular changes via their surface signal transduction pathways. Glioma perivascular niche may thus offer a therapeutic target, especially VEGF secreted by endothelial cells as well as integrins expressed by glioma stem cells.

Growth factors and signaling pathways that affecting vascular proliferation and arborization of the vascular tree: Cancer stem cells secrete a multitude of chemokines and growth factors that induce changes in local tumor stroma including the recruitment and proliferation of BMderived cells to support new vessel development. Notably, VEGF is the most studied factor and is responsible for the coordination of new vessel formation and vascular maintenance. It plays an autocrine and paracrine role within this niche. Additionally, cancer cells may also secrete placental growth factor (PIGF), which promotes adult vasculogenesis by enhancing EPC recruitment and vessel formation at the site of tumor neovascularization [74].

Another characteristic of cancer stem cells is their ability to migrate and infiltrate the surrounding normal tissue. Cancer stem cells acquire mobility by undergo Epithelialmesenchymal transition (EMT). EMT is usually restricted to embryonic development and tissue repair. The activation of this process is crucial for invasive growth and metastasis. Induction of EMT in cancer cells is achieved by a combination of stromal factors, such as HGF, TGF $\beta$, tumor necrosis factor- $\alpha$ and MMPs, and by activating mutations in the RAS proto-oncogene [75-77]. The expression of VEGFA and MMPs, are regulated by hypoxia and pro-inflammatory cytokines. MMPs via their protease activity are required for the degradation of the cellular matrix, a 
prerequisite for vessel sprouting.

The other set of signaling pathways important in this process include signaling proteins activated via TGF and EGF receptors. Signal transducer and activator of transcription3 (STAT3) promotes both wound repair and carcinogenesis [78]. Mice that express a constitutively active form of STAT3 in the epidermis develop skin cancer with a shorter latency; the number and malignancy of the tumors were also increased. STAT3 suppresses re-epithelization thus creating a persistent inflammatory state that resembles a chronic wound and a permissible milieu for tumorigenesis [79]. Re-epithelialization terminates neo-angiogenesis, prunes and normalizes the neoangiomatous arcades within the granulating tissue, resulting in decreased influx of cells and reestablishing a quiescent state. In the case of tumorigenesis it closes the temporal window for cancer stem cells proliferation and initiates tumor involution.

It has been known that STAT-3 is essential for the maintenance of an undifferentiated embryonic stem cells pool and the pluripotency of its constituent cells [80]. The importance of STAT-3 has also been addressed in glioblastoma stem cells (GBM-SC). Small molecule inhibitors (STA-21 and S31-201) or short hairpin RNA reduce GBM-SC proliferation and neurosphere formation [81]. It is noteworthy that PTEN negatively regulates STAT-3 and mTOR resulting in stem cells/cancer stem cells quiescence, and PTEN is mutated or deleted in 44\% and $60 \%$ of GBM patients respectively [82,83]. Hence dysregulation of STAT-3 may be an important event in activation of cancer stem cells.

In the past, Osetopontin has been seen as an important player in the regulation of stem cell renewal particularly at the stem cell niche, it is a negative regulator in the stem cells niche where it helps to limit the size of the stem cell pool [84]. However, the expression of this extracellular matrix associate protein has been linked to tumor progression and metastasis of breast and other solid tumor including GBM. The casual relation of this process relies on the activity of $\mathrm{a}_{\mathrm{v}} \aleph_{3}$ integrin mediated Janus Kinase 2 (JAK-2) phosphorylation [85]. Paradoxically, Osetopontin deregulates stem cells/cancer stem cell quiescence by increased phosphorylation of STAT-3 in an environment where excess $\mathrm{a}_{\mathrm{v}} \S_{3}$ is available. This frees the cancer stem cells from the regulatory restrain imposed by terminal differentiation. This equilibrium with the vascular niche determines the direction of tumor progression or tumor involvement or quiescence.

\section{The function of cancer stem cell vascular niche complexes and future direction}

The cancer stem cells theory of tumorigenesis is intertwined with the theory of dormancy of cancer. Recent study has postulated that cancer cells are under constant scrutiny by the immune system [86]. For cancer stem cells, quiescence might be a protective response to a microenvironment that is hostile to its survival and lacks tumorigenic recruitment signals. It has been shown that immuno-editing can promote epithelial-mesenchymal transition and favor cancer stem cells [87]. The decisive force that propels cancer stem cells to achieve a higher degree of autonomy within the niche remains unclear. It is unlikely to be an isolated stochastic event, rather an integration of multiple random events that are closely linked chronologically which ignites the angiogenic switch and the maturation of the vascular elements. The influx of foreign cells such as circulating monocytic cells and vascular progenitors along with the factors expressed by these cells may have fueled epigenetic changes in the naive cells and altered the cellular matrix (Figure 2). The possibility of aberrant signaling events induced by an altered micro-environment, leading to reversible epigenetic changes in each cell type, underscores the heterogeneity within the developing tumor [88]. This allows abrupt changes in the phenotypic expression of the cancer cells, overwhelming the immuno-surveillance and immuno- 
suppression. It also confers mobility of the cells to prepare for invasion and distant metastasis. Since these epigenetic changes are reversible they may not be easily recognizable in the final tumor. Other factors that may play a pivotal role in this process are loss of contact inhibition, the rise in hypoxic gradient, and increase in interstitial tension within the niche. All of these processes are intimately linked to further epigenetic alternation and play an important role in malignant transformation resulting in quasi-malignant phenotypes.

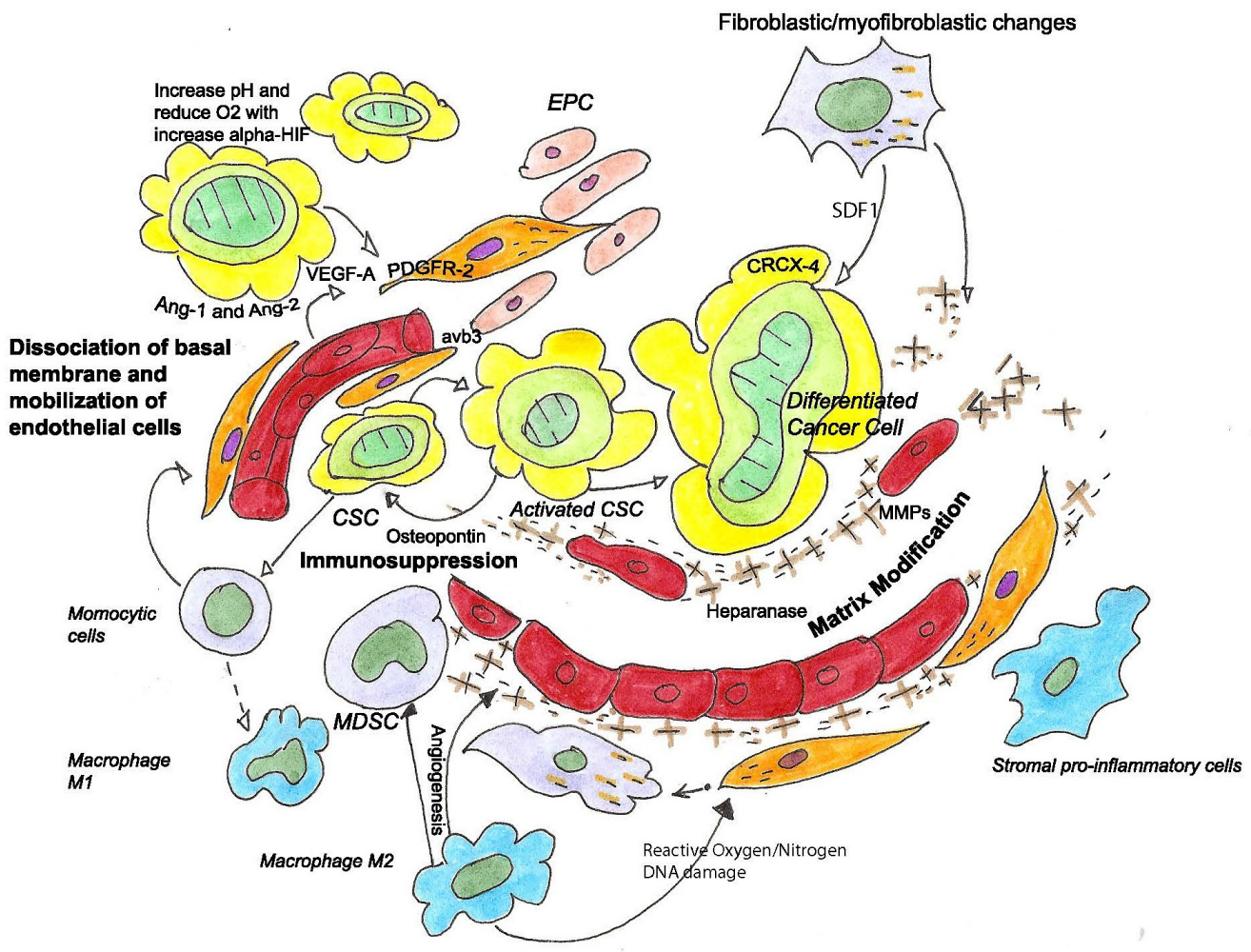

\section{FIGURE 2: Glioma stem cells and vascular niche milieu}

Tumor core are in hypoxic states that causes the increase of HIF1a. This increase in transcription factor HIF1a leads to increased VEGF expression with a consequent increase in endothelial cells and neoangiogenesis. This is aided by the dissociation of the basal lamina via overexpression of MMP and heparanase. This breakdown causes endothelial cells to generate nascent vascular cords that differentiate to functional vasculature. The increased in M2 macrophage leads to increased reactive oxygen and nitrogen species perpetuating the inflammatory process. The migration and differentiation of cancer stem cells to cancer cells is aided by the action of SDF1 released by the fibroblasts that are associated with the tumor.

It is clear that brain tumors especially gliomas are highly dependent on angiogenesis for their growth. This provides an overwhelming rationale for anti-angiogenic approaches for treating these deadly tumors. Recent studies have suggested that anti-angiogenic therapy may indeed be necessary to "normalize" the glioma vasculature for antitumor agents to reach an effective concentration. Most of these agents target the VEGF or PDGF ligand/receptor interaction that target both cancer cells and endothelial cells. Increased doses of anti-angiogenic agents currently in trial could produce complete tumor regression, but such doses are accompanied with immense side effects. Several agents targeting angiogenesis have been approved for use in non-CNS tumor. Multiple drugs are in various stages of trials in glioma and other CNS tumors (Table 2). 


\section{Cureus}

\begin{tabular}{|c|c|c|}
\hline Agent & Phase & Target \\
\hline 2ME2 & Phase II & Inhibits HIF-1a \\
\hline Cediranib & Phase II & VEGFR and PDGFR inhibitor \\
\hline Pazopanib & Phase II & VEGFR and PDGFR inhibitor \\
\hline PTC299 & Phase II & Inhibits VEGF at the post-transcriptionally \\
\hline Sorafenib & Phase I/II & VEGFR, PDGFR and BRAF inhibitor \\
\hline Sunitinib & Phase II & VEGFR and PDGFR Inhibitor \\
\hline Iandutınıb & Phase II & InhibIts PDGFR \\
\hline Vandetanib & Phase I/II & Inhibits VEGFR and EGFR \\
\hline Vatalanib & Phase I/II & VEGFR and PDGFR inhibitor \\
\hline ABT510 & Phase II & Inhibits CD36 receptor \\
\hline Aflibercept & Phase I/II & Decoy receptor for VEGF \\
\hline ATN161 & Phase II & Peptide inhibits integrin a5ß1 \\
\hline Bevacizumab & Phase II/III & Monoclonal antibody binds to VEGF \\
\hline Cilengitide & Phase II/III & RGD synthetic peptide inhibits integrin av $B 3$ and $a_{v} B_{5}$ \\
\hline СТ322 & Phase I & Fibronectin based VEGFR inhibitor \\
\hline Interferon Alfa 2b & Phase II & Inhibits angiogenesis \\
\hline TM601 & Phase I & Peptide binds to Annexin A \\
\hline
\end{tabular}

TABLE 2: Antiangiogenic agents currently at various stages of clinical trial for glioma (peptide/proteins are marked in shaded boxes)

\section{Conclusions}

A vascular niche complex is a permissible environment in which the cellular and humoral elements that support repair and remodeling of healthy tissue have gone disarray. As in wound healing and tissue repair, the side population of tissue stem cells and its associated cells are activated; aided by the influxes of vascular progenitors and inflammatory cells, they rebuild and remodel the injury site and restore normal tissue architecture. In tumorigenesis, the activation and the formation of the cancer stem cells-vascular niche complex is the corollary of having a chance event in which a stem cell with deleterious mutation takes root in an epigenetically corrupted niche. The formation of this permissible milieu is the pre-requisition for cancer stem cells to achieve their malignant potential.

Tumor angiogenesis has long been the target in therapeutic designs; numerous strategies have been formulated to disrupt its formation. With the concept of Cancer stem cell vascular niche 
complex, it may be more beneficial in finding way to "normalize" this milieu by putting more emphasis on the supportive cells and the matrix.

\section{Additional Information}

\section{Disclosures}

Conflicts of interest: In compliance with the ICMJE uniform disclosure form, all authors declare the following: Payment/services info: All authors have declared that no financial support was received from any organization for the submitted work. Financial relationships: All authors have declared that they have no financial relationships at present or within the previous three years with any organizations that might have an interest in the submitted work. Other relationships: All authors have declared that there are no other relationships or activities that could appear to have influenced the submitted work.

\section{Acknowledgements}

Author Contribution: HB and VT prepared the manuscript.

\section{References}

1. Bailey P, Cushing H: A classification of the tumor of the glioma group on a histogenetic basis with a correlated study of prognosis.. JB Lippincott, Philadelphia; 1926.

2. Fukumura D, Duda DG, Munn LL, Jain RK: Tumor microvasculature and microenvironment: novel insights through intravital imaging in pre-clinical models. Microcirculation. 2010, 17:206-225. 10.1111/j.1549-8719.2010.00029.x

3. Naumov GN, Folkman J, Straume O: Tumor dormancy due to failure of angiogenesis: role of the microenvironment. Clin Exp Metastasis. 2009, 26:51-60 . 10.1007/s10585-008-9176-0

4. Holash J, Maisonpierre PC, Compton D, et al: Vessel cooption, regression, and growth in tumors mediated by angiopoietins and VEGF. Science. 1999, 284:1994-1998.

5. Gilbertson RJ, Rich JN: Making a tumour's bed: glioblastoma stem cells and the vascular niche. Nat Rev Cancer. 2007, 7:733-736.

6. Uchida N, Buck DW, He D, et al: Direct isolation of human central nervous system stem cells . Proc Natl Acad Sci USA. 2000, 97:14720-14725.

7. Pollard SM, Yoshikawa K, Clarke ID, et al: Glioma stem cell lines expanded in adherent culture have tumor-specific phenotypes and are suitable for chemical and genetic screens. Cell Stem Cell. 2009, 4:568-580. 10.1016/j.stem.2009.03.014

8. Knudson AG Jr.: Mutation and cancer: statistical study of retinoblastoma . Proc Natl Acad Sci USA. 1971, 68:820-823.

9. Baker SJ, Preisinger AC, Jessup JM, et al: p53 gene mutations occur in combination with 17p allelic deletions as late events in colorectal tumorigenesis. Cancer Res. 1990, 50:7717-7722.

10. Reya T, Morrison SJ, Clarke MF, Weissman IL: Stem cells, cancer, and cancer stem cells. Nature. 2001, 414:105-111.

11. Singh SK, Hawkins C, Clarke ID, et al: Identification of human brain tumour initiating cells . Nature. 2004, 432:396-401.

12. Liang Y, Zhong Z, Huang Y, et al: Stem-like cancer cells are inducible by increasing genomic instability in cancer cells. J Biol Chem. 2010, 285:4931-4940. 10.1074/jbc.M109.048397

13. Shiras A, Chettiar ST, Shepal V, Rajendran G, Prasad GR, Shastry P: Spontaneous transformation of human adult nontumorigenic stem cells to cancer stem cells is driven by genomic instability in a human model of glioblastoma. Stem Cells. 2007, 25:1478-1489.

14. Cheshier SH, Kalani MY, Lim M, Ailles L, Huhn SL, Weissman IL: A neurosurgeon's guide to stem cells, cancer stem cells, and brain tumor stem cells. Neurosurgery. 2009, 65:237-249. 10.1227/01.NEU.0000349921.14519.2A

15. Calabrese C, Poppleton H, Kocak M, et al: A perivascular niche for brain tumor stem cells . Cancer Cell. 2007, 11:69-82.

16. Christensen K, Schrøder HD, Kristensen BW: CD133 identifies perivascular niches in grade IIIV astrocytomas. J Neurooncol. 2008, 90:157-170. 10.1007/s11060-008-9648-8 
17. Kappadakunnel M, Eskin A, Dong J, et al: Stem cell associated gene expression in glioblastoma multiforme: relationship to survival and the subventricular zone. J Neurooncol. 2010, 96:359367. 10.1007/s11060-009-9983-4

18. De Palma M, Naldini L: Role of haematopoietic cells and endothelial progenitors in tumour angiogenesis. Biochim Biophys Acta. 2006, 1766:159-166.

19. Rafii S, Heissig B, Hattori K: Efficient mobilization and recruitment of marrow-derived endothelial and hematopoietic stem cells by adenoviral vectors expressing angiogenic factors. Gene Ther. 2002, 9:631-641.

20. Du R, Lu KV, Petritsch C, et al: HIF1alpha induces the recruitment of bone marrow-derived vascular modulatory cells to regulate tumor angiogenesis and invasion. Cancer Cell. 2008, 13:206-220. 10.1016/j.ccr.2008.01.034

21. Pietras K, Pahler J, Bergers G, Hanahan D: Functions of paracrine PDGF signaling in the proangiogenic tumor stroma revealed by pharmacological targeting. PLoS Med. 2008, 5:19941998. 10.1371/journal.pmed.0050019

22. Vajkoczy P, Blum S, Lamparter M, et al: Multistep nature of microvascular recruitment of ex vivo-expanded embryonic endothelial progenitor cells during tumor angiogenesis. J Exp Med. 2003, 197:1755-1765.

23. Lyden D, Hattori K, Dias S, et al: Impaired recruitment of bone-marrow-derived endothelial and hematopoietic precursor cells blocks tumor angiogenesis and growth. Nat Med. 2001, 7:1194-1201.

24. Gothert JR, Gustin SE, van Eekelen JA, et al: Genetically tagging endothelial cells in vivo: bone marrow-derived cells do not contribute to tumor endothelium. Blood. 2004, 104:1769-1777.

25. Ueno H, Weissman IL: The origin and fate of yolk sac hematopoiesis: application of chimera analyses to developmental studies. Int J Dev Biol. 2010, 54:1019-1031. 10.1387/ijdb.093039hu

26. Choi K: The hemangioblast: a common progenitor of hematopoietic and endothelial cells . J Hematother Stem Cell Res. 2002, 11:91-101.

27. Quirici N, Soligo D, Caneva L, Servida F, Bossolasco P, Deliliers GL: Differentiation and expansion of endothelial cells from human bone marrow CD133(+) cells. Br J Haematol. 2001, 115:186-194.

28. Shyu WC, Lin SZ, Yen PS, Su CY, Chen DC, Wang HJ, Li H: Stromal cell-derived factor-1 alpha promotes neuroprotection, angiogenesis, and mobilization/homing of bone marrow-derived cells in stroke rats. J Pharmacol Exp Ther. '2008, 324:834-849.

29. Moore XL, Lu J, Sun L, Zhu CJ, Tan P, Wong MC: Endothelial progenitor cells' "homing" specificity to brain tumors. Gene Ther. 2004, 11:811-818.

30. Kokovay E, Li L, Cunningham LA: Angiogenic recruitment of pericytes from bone marrow after stroke. J Cereb Blood Flow Metab. 2006, 26:545-555.

31. Nolan DJ, Ciarrocchi A, Mellick AS, et al: Bone marrow-derived endothelial progenitor cells are a major determinant of nascent tumor neovascularization. Genes Dev. 2007, 21:15461558.

32. Guo P, Hu B, Gu W, et al: Platelet-derived growth factor-B enhances glioma angiogenesis by stimulating vascular endothelial growth factor expression in tumor endothelia and by promoting pericyte recruitment. Am J Pathol. 2003, 162-1083-1093:1083-1093.

33. Morikawa S, Baluk P, Kaidoh T, Haskell A, Jain RK, McDonald DM: Abnormalities in pericytes on blood vessels and endothelial sprouts in tumors. Am J Pathol. 2002, 160:985-1000.

34. Bababeygy SR, Cheshier SH, Hou LC, Higgins DM, Weissman IL, Tse VC: Hematopoietic stem cell-derived pericytic cells in brain tumor angio-architecture. Stem Cells Dev. 2008, 17:11-8. 10.1089/scd.2007.0117

35. Song S, Ewald AJ, Stallcup W, Werb Z, Bergers G: PDGFRbeta+ perivascular progenitor cells in tumours regulate pericyte differentiation and vascular survival. Nat Cell Biol. 2005, 7:870-879.

36. Wu X, Hu J, Zhou L, et al: In vivo tracking of superparamagnetic iron oxide nanoparticlelabeled mesenchymal stem cell tropism to malignant gliomas using magnetic resonance imaging. Laboratory investigation. J Neurosurg. 2008, 108:320-329.

37. Ball SG, Shuttleworth CA, Kielty CM: Mesenchymal stem cells and neovascularization: role of platelet-derived growth factor receptors. J Cell Mol Med. 2007, 11:1012-1030.

38. Raesaenen K, Vaheri A: Activation of fibroblasts in cancer stroma . Exp Cell Res. 2010, 316:2713-2722. 10.1016/j.yexcr.2010.04.032

39. Yung YC, Cheshier S, Santarelli JG, Huang Z, Wagers A, Weissman I, Tse V: Incorporation of naive bone marrow derived cells into the vascular architecture of brain tumor. 
Microcirculation. 22004, 11:699-708.

40. Udani VM, Santarelli JG, Yung YC, Wagers AJ, Cheshier SH, Weissman IL, Tse V:

Hematopoietic stem cells give rise to perivascular endothelial-like cells during brain tumor angiogenesis. Stem Cells Dev. 2005, 14:478-486.

41. Conejo-Garcia JR, Benencia F, Courreges MC, et al: Tumor-infiltrating dendritic cell precursors recruited by a beta-defensin contribute to vasculogenesis under the influence of Vegf-A. Nat Med. 2004, 10:950-958.

42. Santarelli JG, Sarkissian V, Hou LC, Veeravagu A, Tse V: Molecular events of brain metastasis . Neurosurg Focus. 2007, 22:1.

43. Santarelli JG, Udani V, Yung YC, et al: Incorporation of bone marrow-derived Flk-1-expressing CD34+ cells in the endothelium of tumor vessels in the mouse brain. Neurosurgery. 2006, 59:374-382.

44. Walkley CR, Olsen GH, Dworkin S, et al: A microenvironment-induced myeloproliferative syndrome caused by retinoic acid receptor gamma deficiency. Cell. 2007, 129:1097-1110.

45. Stout RD, Jiang C, Matta B, Tietzel I, Watkins SK, Suttles J: Macrophages sequentially change their functional phenotype in response to changes in microenvironmental influences. J Immunol. 2005, 175:342-349.

46. Nagaraj S, Gabrilovich DI: Myeloid-derived suppressor cells. Adv Exp Med Biol. 2007, 601:213223.

47. Movahedi K, Guilliams M, Van den Bossche J, et al: Identification of discrete tumor-induced myeloid-derived suppressor cell subpopulations with distinct T cell-suppressive activity. Blood. 2008, 111:4233-4244. 10.1182/blood-2007-07-099226

48. Mishra PJ, Humeniuk R, Medina DJ, et al: Carcinoma-associated fibroblast-like differentiation of human mesenchymal stem cells. Cancer Res. 2008, 68:4331-4339. 10.1158/0008-5472.CAN08-0943

49. Zeine R, Salwen HR, Peddinti R, et al: Presence of cancer-associated fibroblasts inversely correlates with Schwannian stroma in neuroblastoma tumors. Mod Pathol. 2009, 22:950-958. 10.1038/modpathol.2009.52

50. Orimo A, Weinberg RA: Stromal fibroblasts in cancer: a novel tumor-promoting cell type . Cell Cycle. 2006, 5:1597-1601.

51. Jodele S, Blavier L, Yoon JM, DeClerck YA: Modifying the soil to affect the seed: role of stromal-derived matrix metalloproteinases in cancer progression. Cancer Metastasis Rev. 2006, 25:35-43.

52. Ricci-Vitiani L, Pallini R, Biffoni M, et al: Tumour vascularization via endothelial differentiation of glioblastoma stem-like cells. Nature. 2010, 468:824-828. 10.1038/nature09557

53. Wang R, Chadalavada K, Wilshire J, et al: Glioblastoma stem-like cells give rise to tumour endothelium. Nature. 2010, 468:829-833. 10.1038/nature09624

54. Bolos V, Blanco M, Medina V, Aparicio G, Diaz-Prado S, Grande E: Notch signalling in cancer stem cells. Clin Transl Oncol. 2009, 11:11-9.

55. Warburg O: On the origin of cancer cells . Science. 1956, 123:309-314.

56. DeBerardinis RJ, Lum JJ, Hatzivassiliou G, Thompson CB: The biology of cancer: metabolic reprogramming fuels cell growth and proliferation. Cell Metab. 2008, 7:11-20.

10.1016/j.cmet.2007.10.002

57. Bonnet S, Archer SL, Allalunis-Turner J, et al: A mitochondria-K+ channel axis is suppressed in cancer and its normalization promotes apoptosis and inhibits cancer growth. Cancer Cell. 2007, 11:37-51.

58. MacKenzie ED, Selak MA, Tennant DA, et al: Cell-permeating alpha-ketoglutarate derivatives alleviate pseudohypoxia in succinate dehydrogenase-deficient cells. Mol Cell Biol. 2007, 27:3282-3289.

59. Kim JW, Dang CV: Cancer's molecular sweet tooth and the Warburg effect. Cancer Res. 2006, 66:8927-8930.

60. Gatenby RA, Gillies RJ: Why do cancers have high aerobic glycolysis? . Nat Rev Cancer. 2004, 4:891-899.

61. Michelakis ED, Sutendra G, Dromparis P, et al: Metabolic modulation of glioblastoma with dichloroacetate. Sci Transl Med. 2010, 2:31-34. 10.1126/scitranslmed.3000677

62. Kamoun WS, Chae SS, Lacorre DA, et al: Simultaneous measurement of RBC velocity, flux, hematocrit and shear rate in vascular networks. Nat Methods. 2010, 7:655-660. 


\subsection{8/nmeth.1475}

63. Sun A, Hou L, Prugpichailers T, et al: Firefly luciferase-based dynamic bioluminescence imaging: a noninvasive technique to assess tumor angiogenesis. Neurosurgery. 2010, 66:751757. 10.1227/01.NEU.0000367452.37534.B1

64. Soeda A, Park M, Lee D, et al: Hypoxia promotes expansion of the CD133-positive glioma stem cells through activation of HIF-1alpha. Oncogene. 2009, 28:3949-3959.

10.1038/onc.2009.252

65. Li Z, Bao S, Wu Q, et al: Hypoxia-inducible factors regulate tumorigenic capacity of glioma stem cells. Cancer Cell. 2009, 15:501-513. 10.1016/j.ccr.2009.03.018

66. Lee $\mathrm{OH}, \mathrm{Xu}$ J, Fueyo J, et al: Angiopoietin-2 decreases vascular endothelial growth factor expression by modulating HIF-1 alpha levels in gliomas. Oncogene. 2008, 27:1310-1314.

67. Tse V, Xu L, Yung YC, Santarelli JG, Juan D, Fabel K, Silverberg G, Harsh G 4th: The temporalspatial expression of VEGF, angiopoietins-1 and 2, and Tie-2 during tumor angiogenesis and their functional correlation with tumor neovascular architecture. Neurol Res. 2003, 25:729738.

68. Melder RJ, Koenig GC, Witwer BP, Safabakhsh N, Munn LL, Jain RK: During angiogenesis, vascular endothelial growth factor and basic fibroblast growth factor regulate natural killer cell adhesion to tumor endothelium. Nat Med. 1996, 25:992-997.

69. Kaplan RN, Rafii S, Lyden D: Preparing the "soil": the premetastatic niche. Cancer Res. 2006, 66:11089-11093.

70. Bergers G, Brekken R, McMahon G, et al: Matrix metalloproteinase-9 triggers the angiogenic switch during carcinogenesis. Nat Cell Biol. 2000, 2:737-744.

71. Feng W, McCabe NP, Mahabeleshwar GH, Somanath PR, Phillips DR, Byzova TV: The angiogenic response is dictated by beta3 integrin on bone marrow-derived cells. J Cell Biol. 2008, 183:1145-1157. 10.1083/jcb.200802179

72. Lathia JD, Gallagher J, Heddleston JM, et al: Integrin alpha 6 regulates glioblastoma stem cells. Cell Stem Cell. 2010, 6:421-432. 10.1016/j.stem.2010.02.018

73. Reardon DA, Fink KL, Mikkelsen T, et al: Randomized phase II study of cilengitide, an integrin-targeting arginine-glycine-aspartic acid peptide, in recurrent glioblastoma multiforme. J Clin Oncol. 2008, 26:5610-5617. 10.1200/JCO.2008.16.7510

74. Kerber M, Reiss Y, Wickersheim A, et al: Flt-1 signaling in macrophages promotes glioma growth in vivo. Cancer Res. 2008, 68:7342-7351. 10.1158/0008-5472.CAN-07-6241

75. Roberts WG, Delaat J, Nagane M, Huang S, Cavenee WK, Palade GE: Host microvasculature influence on tumor vascular morphology and endothelial gene expression. Am J Pathol. 1998, 153:1239-1248.

76. Mikheeva SA, Mikheev AM, Petit A, et al: TWIST1 promotes invasion through mesenchymal change in human glioblastoma. Mol Cancer. 2010, 9:194. 10.1186/1476-4598-9-194

77. Horiguchi K, Shirakihara T, Nakano A, Imamura T, Miyazono K, Saitoh M: Role of Ras signaling in the induction of snail by transforming growth factor-beta. J Biol Chem. 2009, 284:245-253. 10.1074/jbc.M804777200

78. Dauer DJ, Ferraro B, Song L, Yu B, Mora L, Buettner R, Enkemann S, Jove R, Haura EB: Stat3 regulates genes common to both wound healing and cancer. Oncogene. 2005, 24:3397-3408.

79. Iliopoulos D, Jaeger SA, Hirsch HA, Bulyk ML, Struhl K: STAT3 activation of miR-21 and miR181b-1 via PTEN and CYLD are part of the epigenetic switch linking inflammation to cancer. Mol Cell. 2010, 39:493-506. 10.1016/j.molcel.2010.07.023

80. Ying QL, Nichols J, Chambers I, Smith A: BMP induction of Id proteins suppresses differentiation and sustains embryonic stem cell self-renewal in collaboration with STAT3. Cell. 2003, 115:281-292.

81. Sherry MM, Reeves A, Wu JK, Cochran BH: STAT3 is required for proliferation and maintenance of multipotency in glioblastoma stem cells. Stem Cells. 2009, 27:2383-2392. 10.1002/stem.185

82. Zheng H, Ying H, Yan H, et al: p53 and Pten control neural and glioma stem/progenitor cell renewal and differentiation. Nature. 1008, 445:1129-1133. 10.1038/nature07443

83. Parsons DW, Jones S, Zhang X, et al: An integrated genomic analysis of human glioblastoma multiforme. Science. 2008, 321:1807-1812. 10.1126/science.1164382

84. Stier S, Ko Y, Forkert R, et al: Osteopontin is a hematopoietic stem cell niche component that negatively regulates stem cell pool size. J Exp Med. 2005, 201:1781-1791.

85. Behera R, Kumar V, Lohite K, Karnik S, Kundu GC: Activation of JAK2/STAT3 signaling by 


\section{Cureus}

osteopontin promotes tumor growth in human breast cancer cells. Carcinogenesis. 2010, 31:192-200. 10.1093/carcin/bgp289

86. Dunn GP, Bruce AT, Ikeda H, Old LJ, Schreiber RD: Cancer immunoediting: from immunosurveillance to tumor escape. Nat Immunol. 2002, 3:991-998.

87. Reiman JM, Knutson KL, Radisky DC: Immune promotion of epithelial-mesenchymal transition and generation of breast cancer stem cells. Cancer Res. 2010, 70:3005-3008. 10.1158/0008-5472.CAN-09-4041

88. Clarke MF, Dick JE, Dirks PB, et al: Cancer stem cells--perspectives on current status and future directions: AACR Workshop on cancer stem cells. Cancer Res. 2006, 66: 